\title{
GRN mutation in a patient with a behavioral variant of frontotemporal lobar degeneration (bvFTD)
}

\author{
Sylwia Walczysková ${ }^{1}$, Pavel Ressner², Šárka Hilscherová ${ }^{1}$, Jaroslav Kotlas ${ }^{3}$, Jiři Konrád ${ }^{4}$, Věnceslava Svobodová5 \\ ${ }^{1}$ Department of Medical Genetics, University Hospital Ostrava, Ostrava, ${ }^{2}$ Neurology Clinic, University Hospital Ostrava, Ostrava, \\ ${ }^{3}$ Institute of Biology and Medical Genetics, General University Hospital, Prague, ${ }^{4}$ Psychiatric Hospital Havlíčkův Brod, \\ Gerontopsychiatry, Havlíčkův Brod, ${ }^{5}$ KlinNeuro Prague Ltd, Neurology, Physiotherapy, Neurosurgery, Prague, Czech Republic
}

\begin{abstract}
The clinical spectrum of frontotemporal lobar degeneration (FTLD) is characterized by personality changes, language impairment, and executive function deficits. About $40 \%$ of FTLD cases have a family history of the disease, and the GRN gene is currently the most frequent genetic determinant. In cases of inherited FTLD with GRN mutations, parkinsonism is often an early sign due to greater grey matter atrophy in the caudate nucleus and bilateral atrophy in the thalamus. We investigated a female patient with signs of frontotemporal lobe atrophy and unilateral caudate nucleus atrophy on MRI. DNA was isolated from peripheral blood leukocytes and tested for GRN gene mutations. A pathogenic splice donor site mutation, c.708+1G>A, was found in the GRN gene. MRI showed unilateral caudate nucleus atrophy. This report extends the evidence for phenotypic and neuropathological heterogeneity in FTLD spectrum disorders due to splicing mutations in the GRN gene.
\end{abstract}

Key words: caudate nucleus atrophy, frontotemporal dementia, GRN gene, splicing mutation.

\section{Introduction}

Patients $<65$ years old with presenile dementia make up $5-15 \%$ of all patients with dementia. Frontotemporal lobar degeneration (FTLD) is the second most common cause of early onset dementia after Alzheimer's disease. Frontotemporal lobar degeneration is a highly heritable disorder, with about 25-50\% of patients having a positive family history and about $10 \%$ showing clear autosomal-dominant inheritance, even though a genetic cause can be demonstrated in less than $20 \%$ of patients [2]. This neurodegenerative disorder shows genetic and pathological heterogeneity, is characterized by behavior and language distur- bances, and is associated with variable frontal, temporal, and basal ganglia atrophy with neuronal loss and gliosis $[2,16]$. The redefined clinical criteria recognize different phenotypes based on the clinical symptoms at presentation, including a possible behavioral variant of FTLD (bvFTD), two language variants, primary progressive nonfluent aphasia (PNFA), and a semantic variant. Notably, all variants can overlap with atypical Parkinsonian disorders such as progressive supranuclear palsy and corticobasal degeneration $[5,12,14]$.

Frontotemporal lobar degeneration has been linked to mutations in seven genes (TARDBP, FUS, MAPT, 
GRN, VCP, CHMP2B, and C9ORF72), three of which account for the vast majority of familial FTLD cases and for a proportion of sporadic FTLD cases: the MAPT gene (microtubule-associated protein Tau), the GRN gene (also known as PGRN, progranulin), and the C9ORF72 gene (expanded hexanucleotide repeat in a non-coding region of chromosome 9 open reading frame 72 ). About $95 \%$ of individuals who are diagnosed with FTLD with a pathogenic mutation in the GRN gene (FTLD-GRN) have an affected parent. The age of clinical onset varies widely in GRN mutation carriers, even within the same family, and it is currently not possible to predict the exact age of disease onset in an asymptomatic individual. The proportion of cases caused by de novo mutation is unknown but is estimated to be $5 \%$ or less [11] The GRN gene encodes a secreted multifunctional growth factor that is involved in a wide range of biological processes, such as development, wound repair, and the immune response, among others $[7,16]$. In the FTD patient population, the GRN mutation frequency ranges from $5 \%$ to $26 \%$, and FTLD-GRN is inherited in an autosomal dominant manner. Pathogenic mutations in the GRN gene are typically loss-of-function, nonsense, frame shift, or splice-site mutations that lead to GRN haploinsufficiency (null mutations). Studies have shown that the GRN levels in cerebrospinal fluid (CSF) do not differ in patients with a variety of types of primary neurodegenerative dementias. However, the plasma PGRN level (up to $35-75 \%$ reduction in mutation carriers with respect to controls), which can be used to detect GRN mutation carriers, is one of the best examples of a reliable plasma biomarker in neurodegenerative diseases $[6,17]$. Subcortical and deep cortical involvement is a key feature of FTLD and especially of GRN-related disease, which shows a significantly higher degree of atrophy bilaterally in the caudate nucleus and thalamus compared to FTLD patients without a GRN mutation. This may explain the parkinsonism that is frequently associated with the disease even in its early stages. There are some case reports describing the loss of pigmented neurons from the substantia nigra $[9,15,19]$. Here we describe a case of a patient with a rare splicing mutation in the $G R N$ gene and unilateral caudate nucleus atrophy.

\section{Case report}

The patient was a right-handed Caucasian woman (III-2) with 18 years of formal education who began to exhibit memory and cognitive impairment, behavioral changes, and slight language disturbances (word finding difficulties) in 2005 when she was 59 years old. There was a strong family history of dementia in the proband's family (Fig. 1A); specifically, her father (II-1) (deceased at the age of 72 years) and grandmother (I-2) had histories of dementia, as reported by relatives. The woman had 3 sons (IV-1 IV-3), the oldest born in 1974; to date, all are healthy. The proband was otherwise healthy (i.e. no somatic disorders), with a normal full blood count and metabolic, renal, and hepatic function. She had no history of alcohol or drug abuse, not even nicotinism.

In 2005, the proband's family began to notice behavioral changes, such as wandering, instead of going to the work (finally that was the reason for her dismissal), she began to be dissocial (unsociable and indecent in her behavior) and insensitive to the others, and she began telling lies profusely. She exhibited mild executive function disturbances, began to have problems with cooking (she was cooking from rotten or unsuitable ingredients). She reduced food intake due to lack of appetite. At that time she had found herself healthy and refused to visit any physician. She also became forgetful, language problems appeared, she had difficulties finding words and repeated sentences and phrases (perseverations). She repeatedly got lost in her hometown. She was unaware that she was having these issues (anosognosia).

The patient worked as a teacher until 2008. In 2010, her behavior worsened (unreasonable conduct), and she began running away from home with getting lost in the city. At home and lately in the hospital she was walking back and forth permanently in the corridor and opening/closing doors and drawers repetitively. She was not able to do activities of daily living (dressing, self-care, cooking, gadget use), began to lose social inhibition (changing clothes at a parking place abroad), she lost insight. On the basis of clinical symptoms she met criteria for bvFTD diagnosis.

Psychological testing performed in 2010 showed the following scores: MMSE, 26/30; Raven's Progressive Matrices IQ, 117; Wechsler Memory Test MIQ, 77; in Clock Drawing Test the numbers were written correctly, only handed in a slightly incorrect position and, she was unable to distinguish accurately the hour and minute hand. In 2011, her speech became very poor and telegraphic, and she was unable to recall words and phrases, she used agrammatisms, words in wrong and senseless combinations (phonemic paraphasia), 
A

I

I

III

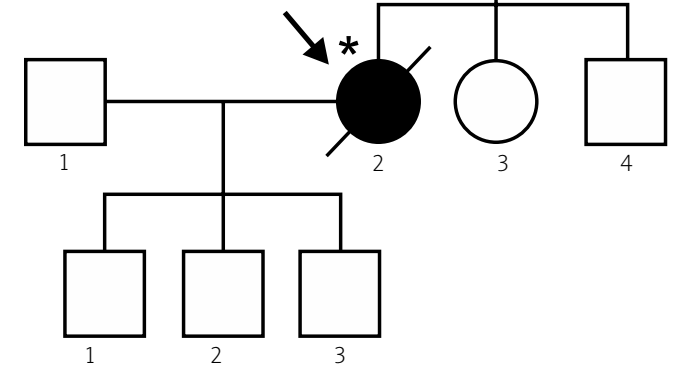

B

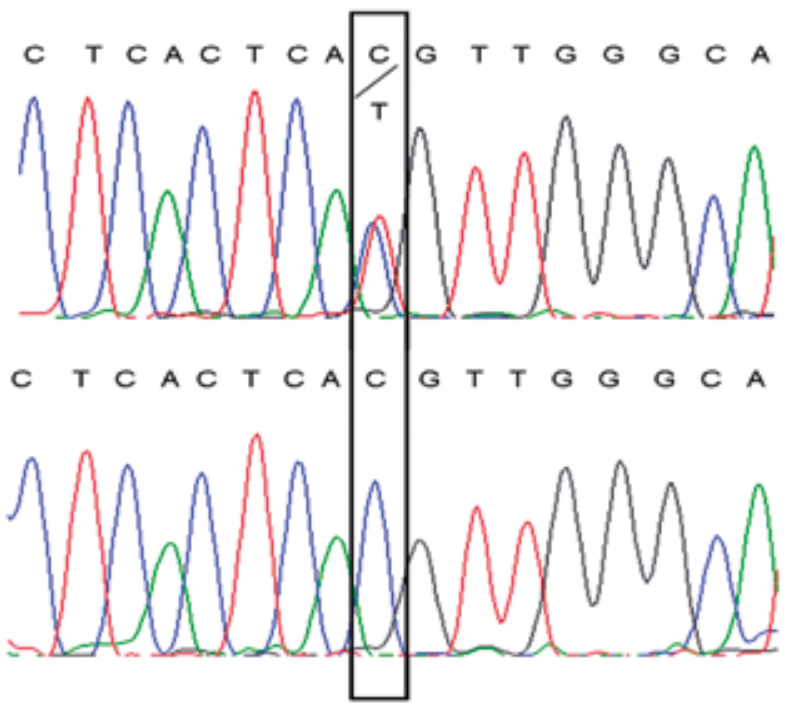

Fig. 1. A) The pedigree of the Czech family showing the inheritance of frontotemporal dementia. Affected individuals are shown as filled symbols and the arrow points to the proband. B) Reverse DNA sequence fragment of the GRN gene. The upper panel shows the pathogenic mutation c.708+1G>A in intron 7, and the lower panel shows the normal sequence.

without dysarthria, with accented perseverations, losing ability to write and read, then she lost capability of forming sentences, in a logopedic exam she was diagnosed as expressive non-fluent aphasia (Broca aphasia). An MRI of her brain revealed left frontotemporal atrophy with ventricular dilatation that was more prominent in the temporal region with atrophy of hippocampus and insula and left caudate nucleus atrophy (Fig. 2).

Over the next year, the speech problems were profusely pronounced and there was a clinical overlap with progressive non-fluent aphasia (nfvPAA). She could understand only very simple instructions, and, for example, she stood up and walked away under the directions. She also had urinary and fecal incontinence.

In 2012, she lost the ability of spontaneous speech; she could only say simple phrases like "You go", and she gradually became mute. In 2013, she needed complete personal care and a nasogastric tube feeding towards the end of the disease, in 2015. She passed away at the age of 69 years from pneumonia related to her disorder. The disease duration was about 8 years.

Two of the woman's three sons were interested in presymptomatic DNA testing and underwent extensive genetic counseling.

\section{Material and methods}

Genomic DNA was extracted from the patient's whole blood using standard procedures. Informed consent to genetic analysis for diagnostic and research purposes was obtained from the patient's legal guardian. Due to the clinical course and strong family history of the disease, we performed mutation analysis of the PSEN1, PSEN2, APP (exons 16, 17 only) and GRN genes. All coding exons and flanking sequences of these genes were amplified using primers, which are available upon request. The amplified fragments were sequenced in both directions using standard protocols and the BigDye Terminator v3.1 Cycle Sequencing Kit and an ABI PRISM3130 genetic analyzer. The sequences were analyzed using SeqScape ${ }^{\circledR}$ software v2.5 (Applied Biosystems, Life Technologies, Carlsbad, CA, USA); NCBI Ref. sequence NM_002087.3. The available brain MRI exams (Magnetom Avanto 1,5 T; Siemens) were analyzed visually. There were 2 independent DNA samples from two sons of the proband available for testing (with informed consent) and for potential confirmation of the mutation.

\section{Results}

Sequence analysis of the patient's GRN gene identified a pathogenic splice donor site mutation, 

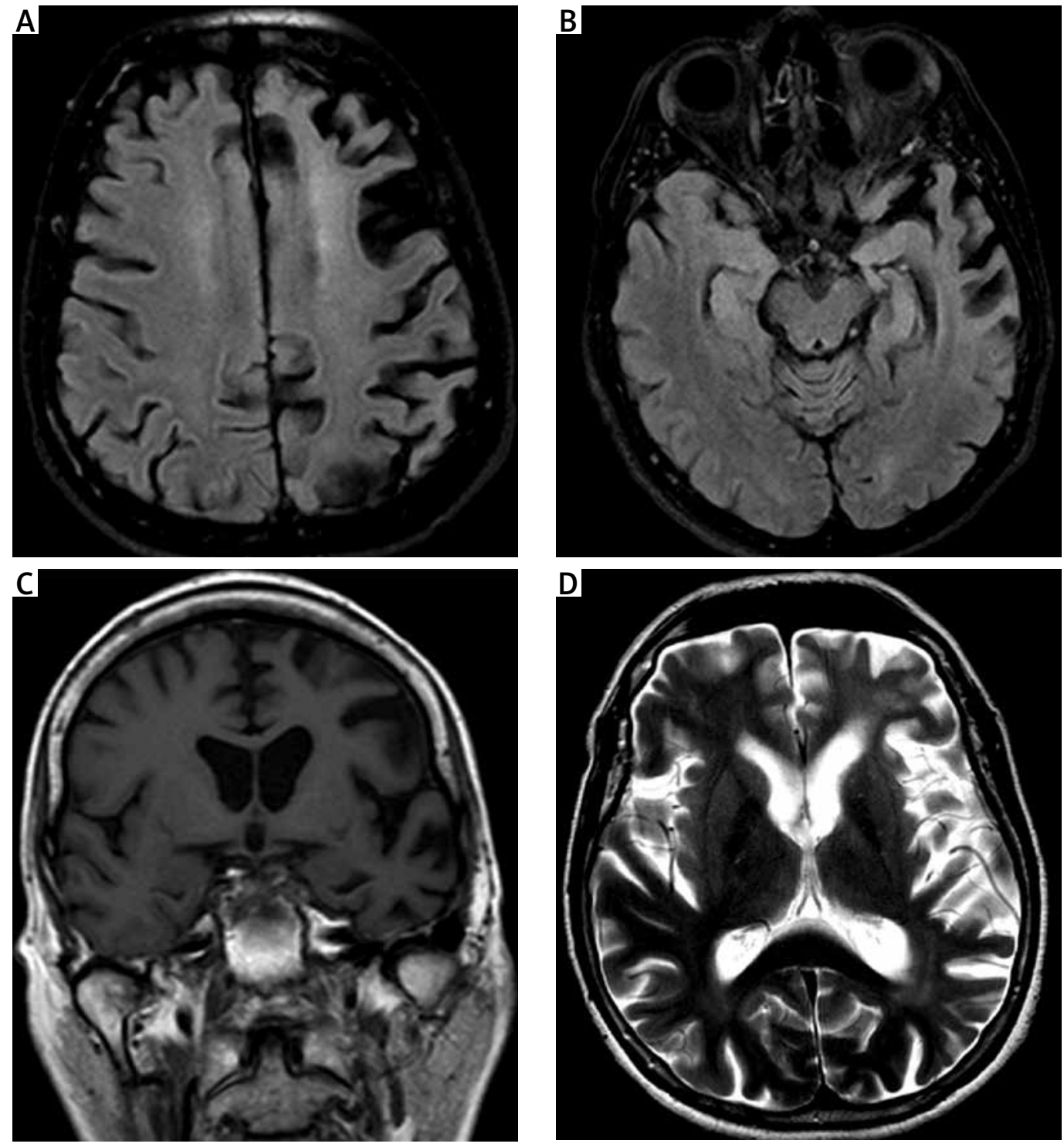

Fig. 2. Magnetic resonance imaging of the patient at 64 years of age. A) FLAIR parietal and frontal atrophy. B) Temporal atrophy that is more marked on the left side. C) T1 coronal image. D) T2 caput caudate nucleus atrophy on the left side.

c. $708+1 \mathrm{G}>\mathrm{A}$, in intron 7 (Fig. 1B). The mutation was heterozygous and was predicted to have dramatic consequences on the maturation of GRN mRNA, leading to the removal of exon 7 and thus creating a frame shift that would result in a truncated protein half of its normal length (protein level, p.Val200GlyfsX18) [16].

\section{Discussion}

Here we report a patient with a pathogenic splice site mutation c.708+1G>A in the GRN gene. This intro- nic mutation was described previously by Masellis et al. (2006) in 2 of 12 siblings that were been affected by corticobasal syndrome (CBS). That report described the evolution of the patients' dementia, motor decline (including rigidity, dystonia, and apraxia), cortical sensory loss, visuospatial dysfunction, behavioral changes, and extrapyramidal features. The caudate nucleus and putamen were atrophic, while the hippocampus was normal in size. Although both family members were diagnosed with CBS, their clinical courses showed important differences. Notably, there 
was no history of dementia or parkinsonism in either parent. Another report described another French family of Caucasian origin with 2 affected members but no family history of the disease [17]. The signs of disease onset included tachyphemia and behavioral disorders such as apathy. In addition, the two had attention disorders, impulsivity, joviality, memory deficits, tachyphemia, and reduction of spontaneous language. These cases were considered a behavioral type of FTLD. The mean age at disease onset in both of these families was at 53-72 years old, and the mean disease duration was about 2-8 years according to the Alzheimer Disease \& Frontotemporal Dementia Mutation database.

Researchers and healthcare providers often consider patterns such as repetitive locomotion following circuitous path or back and forth, as well as random ambulation to be aimless and dementiarelated behavioral disturbances [4]. Some of these patterns are associated with running away, wandering or getting lost [4]. Our proband had a family history of dementia and showed personality and language deterioration along with attempts of running away from home and stereotypic behavior (repetitive walking back and forth, opening the doors and drawers in the corridor). Otherwise she had no specific health problems.

Volumetric studies that compared the rate of brain atrophy in FTD-GRN versus FTD caused by mutations in the MAPT gene (FTD-MAPT) showed that individuals with pathogenic $G R N$ variants have a higher rate (3.5\% vs. $2.4 \%$ per year) of whole-brain atrophy that is more asymmetrically pronounced than those with MAPT-related FTLD [20]. The 2010 brain MRI examination of our proband revealed not only asymmetric involvement with pronounced brain atrophy of the left temporal and frontal lobes and asymmetric caudate nucleus atrophy, which is in agreement with the literature $[3,14]$, but also revealed greatly pronounced caudate nucleus and gyrus rectus atrophy on the left side, with virtually normal findings for the right caudate nucleus, and it would have been very interesting to follow the changes in asymmetry over time. Such a finding appears to be, according to some authors, a typical finding in bvFTD [8]. However, the asymmetric atrophy of the left hemisphere with predominance in temporal and frontal lobes, hippocampi and insula including subcortical atrophy with basal ganglia and the large atrophy of the left caudate nucleus indi- cate the diagnosis of bvFTD in our case. The shape deflation of the left caudate nucleus, which corresponded to afferent connections from the dorsolateral prefrontal mediofrontal/anterior cingulate and orbitofrontal cortex, correlated with worsening disease severity [14]. Our proband with dementia and pathogenic mutation in the GRN gene and a clinical manifestation of the disease fulfilled the diagnostic criteria for bvFTD.

Five years after the disease onset, according to psychological and logopedic examinations, there was a clear progress in speech impairment with agrammatisms, anomia and phonemic paraphasia, later on with speech fluency impairment - almost no spontaneous speech, she was only repeating words sporadically. This was in agreement with progressive non-fluent aphasia (non-fluent PPA) (nfvPPA). Such a course may suggest that types of FTD can overlap in certain stages of disease.

In a report by Harris et al. (2016) it was found that over $90 \%$ of patients with FTLD pathology exhibited a combination of at least one behavioral and one language feature [10]. It would have been interesting to repeat the MRI, but it was not possible due to the death of the patient in 2015. In the above reports by Masellis et al. (2006) and Le Ber et al. (2008), the tested patients were diagnosed with behavioral variants of FTLD. Our patient showed the typical signs of this variant in the early stages of the disease, though the later speech problems suggested the progressive nonfluent aphasia variant. In relation to these initial symptoms, we consider our patient as probable bvFTD. There might be some modifier genes and/or environmental influences, epigenetic factors, ethnicity that prevail over a particular manifestation of one variant of FTLD over another.

In conclusion, this case report highlights the variety of MRI characteristics that are found in patients with frontotemporal dementia and the c.708+1G>A mutation in the GRN gene. The age of clinical onset and survival can vary widely in $G R N$ mutation carriers, even within the same family [1]. A precise diagnosis must be made through genetic testing.

\section{Acknowledgements}

This work was supported by an institutional grant from the Ministry of Health of the Czech Republic (No. 2 RVO-FNOs/2013). 


\section{Disclosure}

\section{Authors report no conflict of interest.}

\section{References}

1. Armstrong RA. Survival in the pre-senile dementia frontotemporal lobar degeneration with the TDP-43 proteinopathy: effects of genetic, demographic and neuropathological variables. Folia Neuropathol 2016; 24: 137-148.

2. Benussi A, Padovani A, Borroni B. Phenotypic heterogeneity of monogenic frontotemporal dementia. Front Aging Neurosci 2015; 7: 171.

3. Buhour MS, Doidy F, Laisney M, Pitel AL, de La Sayette V, Viader F, Eustache F, Desgranges B. Pathophysiology of the behavioral variant of frontotemporal lobar degeneration: a study combining MRI and FDG-PET. Brain Imaging Behav 2016; 1-13.

4. Cipriani G, Lucetti C, Nuti A, Danti S. Wandering and dementia. Psychogeriatrics 2014; 14: 135-142.

5. Dewer B, Rogers P, Ricketts J, Mukonoweshuro W, Zeman A. The radiological diagnosis of frontotemporal dementia in everyday practice: an audit of reports, review of diagnostic criteria, and proposal for service improvement. Clin Radiol 2016; 71 40-47.

6. Finch N, Baker M, Crook R, Swanson K, Kuntz K, Surtees R, Bisceglio G, Rovelet-Lecrux A, Boeve B, Petersen RC, Dickson DW, Younkin SG, Deramecourt V, Crook J, Graff-Radford NR, Rademakers R. Plasma progranulin levels predict progranulin mutation status in frontotemporal dementia and asymptomatic family members. Brain 2009; 132: 583-591.

7. Fontana F, Siva K, Denti MA. A network of RNA and protein interactions in frontotemporal dementia. Front Mol Neurosci 2015; 8: 9

8. Frings L, Yew B, Flanagan E, Lam BYK, Hüll M, Huppertz J, Hodges JR, Hornberger M. Longitudinal grey and white matter changes in frontotemporal dementia and Alzheimer's disease. PLoS Ona 2014; 9: e90814.

9. Gijselinck I, Van Broeckhoven C, Cruts M. Granulin mutations associated with frontotemporal lobar degeneration and related disorders: An update. Hum Mutat 2008; 29: 1373-1386.

10. Harris JM, Jones M, Gall C, Richardson AMT, Neary D, du Plessis D, Pal P, Mann DMA, Snowden JS, Thompson JC. Co-Occurrence of Language and Behavioural Change in Frontotemporal Lobar Degeneration. Dement Geriatr Cogn Disord Extra 2016; 6: 205-213.

11. Hsiung GYR, Feldman HH. GRN-related frontotemporal dementia. In: GeneReviews ${ }^{\circledast}$ [Internet]. Pagon RA et al. (eds.). University of Washington, Seattle 2007; pp. 1993-2016.

12. Lashley T, Rohrer JD, Mead S, Revesz T. Review: an update on clinical, genetic and pathological aspects of frontotemporal lobar degenerations. Neuropathol Appl Neurobiol 2015; 41: 858-881.

13. Le Ber I, Camuzat A, Hannequin D, Pasquier F, Guedj E, RoveletLecrux A, Hahn-Barma V, van der Zee J, Clot F, Bakchine S, Puel M, Ghanim M, Lacomblez L, Mikol J, Deramecourt V, Lejeune P, de la Sayette V, Belliard S, Vercelletto M, Meyrignac Ch, Van Broeckhoven Ch, Lambert JCh, Verpillat P, Campion D, Habert MO, Dubois B, Brice A. Phenotype variability in progranulin muta- tion carriers: a clinical, neuropsychological, imaging and genetic study. Brain 2008; 131: 732-746.

14. Macfarlane MD, Jakabek D, Walterfang M, Vestberg S, Velakoulis D, Wilkes FA, Nilsson C, van Westen D, Looi JC, Santillo AF. Striatal atrophy in the behavioral variant of frontotemporal dementia: correlation with diagnosis, negative symptoms and disease severity. PLoS One 2015; 10: e0129692.

15. Mackenzie IRA, Baker M, Pickering-Brown S, Hsiung GYR, Lindholm C, Dwosh E, Gass J, Cannon A, Rademakers R, Hutton M, Feldman $\mathrm{HH}$. The neuropathology of frontotemporal lobar degeneration caused by mutations in the progranulin gene. Brain 2006; 129: 3081-3090.

16. Masellis M, Momeni P, Meschino W, Heffner Jr R, Elder J, Sato Ch, Liang Y, St George-Hyslop P, Hardy J, Bilbao J, Black S, Rogaeva E. Novel splicing mutation in the progranulin gene causing familial corticobasal syndrome. Brain 2006; 129: 3115-3123.

17. Morenas-Rodríguez E, Cervera-Carles L, Vilaplana E, Alcolea D, Carmona-Iragui M, Dols-Icardo O, Ribosa-Nogué R, MuñozLlahuna L, Sala I, Sánchez-Saudinós MB, Blesa R, Clarimón J, Fortea J, Lleó A. Progranulin protein levels in cerebrospinal fluid in primary neurodegenerative dementias. J Alzheimers Dis 2016; 50: 539-546.

18. Po K, Leslie FVC, Gracia N, Bartley L, Kwok JBJ, Halliday GM, Hodges JR, Burrell JR. Heritability in frontotemporal dementia: more missing pieces? J Neurol 2014; 261: 2170-2177.

19. Premi E, Garibotto V, Gazzina S, Formenti A, Archetti S, Gasparotti R, Padovani A, Borroni B. Subcortical and deep cortical atrophy in frontotemporal dementia due to granulin mutations. Dement Geriatr Cogn Dis Extra 2014; 4: 95-102.

20. Whitwell JL, Weigand SD, Gunter JL, Boeve BF, Rademakers R, Knopman DS, Wszolek ZK, Petersen RC, Jack CR, Josephs KA. Trajectories of brain and hippocampal atrophy in FTD with mutations in MAPT or GRN. Neurology 2011; 77: 393-398. 